\title{
Preparation and Property of Xylan/Poly(Methacrylic Acid) Semi-Interpenetrating Network Hydrogel
}

\author{
Xiao-Feng Sun, Yang Feng, Xiaodi Shi, and Yaxiong Wang \\ MOE Key Laboratory of Applied Physics and Chemistry in Space, College of Science, Northwestern Polytechnic University, \\ Xian 710072, China \\ Correspondence should be addressed to Xiao-Feng Sun; xf001sn@nwpu.edu.cn
}

Received 6 November 2015; Revised 19 February 2016; Accepted 3 March 2016

Academic Editor: Jelena Jovanovic

Copyright (C) 2016 Xiao-Feng Sun et al. This is an open access article distributed under the Creative Commons Attribution License, which permits unrestricted use, distribution, and reproduction in any medium, provided the original work is properly cited.

Xylan/poly(methacrylic acid) semi-interpenetrating network hydrogels were synthesized, and these hydrogels were fabricated using various ratios of xylan and methacrylic acid and cross-linked by $\mathrm{N}, \mathrm{N}^{\prime}$-methylenebisacrylamide. The chemical structure and morphology of the prepared hydrogels were characterized by FT-IR spectroscopy and SEM analysis. The porous structure of the prepared hydrogels was found, and the interconnected porous channels increased when the content of xylan was increased in the hydrogels. The hydrogels presented excellent $\mathrm{pH}$ sensitivity and swelling reversibility. The dynamic swelling kinetics were also studied, and all obtained results indicated that the prepared xylan/poly(methacrylic acid) semi-IPN hydrogel could be used in biomedical fields, especially for drug release.

\section{Introduction}

Hydrogel has a three-dimensional network structure, and it can absorb water but cannot dissolve in water $[1,2]$. Because of its excellent swelling property and multiresponses on environmental stimuli, hydrogel has attracted considerable research interest in many fields, especially in drug controlled release [3-6], enzyme immobilization [7, 8], tissue engineering $[9,10]$, cell culture $[11,12]$, wastewater treatment, and so on [13-15]. Depending on the chemical composition of hydrogel, it is classified into two categories: one is synthetic polymer-based hydrogel that was mainly synthesized from monomers or synthetic polymers such as poly(methacrylic acid); the other is the hydrogel that was prepared from natural polymer, for instance, cellulose, chitosan, alginate, gelatin, konjac glucomannan, guar gum, and other natural polymers $[4,6,8,9,16,17]$. Hydrogel made of poly(methacrylic acid) is an ionizable hydrogel which has been studied for controlled drug release [18], but it is not biodegradable. Because of the excellent biodegradability and biocompatibility of natural polymers, the preparation of these hydrogels becomes a hot research topic. In particular, the hydrogel based on natural polysaccharide can be used in the field of the controlled drug release. Interpenetrating polymer networks hydrogel based on konjac glucomannan and poly(methacrylic acid) had been prepared for controlled drug release [18].

Xylan is second only to cellulose as most prevalent polysaccharide in nature. It possesses $\beta$ - $(1,4)$ linked xylose units as the backbone and sugar- and $O$-acetyl groups as substitutes, and it accounts for $25-35 \%$ of the dry weight of plant cells; therefore, xylan is a rich, inexhaustible, and renewable polymer [19]. Xylan has biodegradable property and biocompatibility and possesses inhibitory actions on mutagenicity activity and antiphlogistic effects and immune functions [20-22]. In previous studies, we have studied the separation and chemical structure of xylan $[23,24]$, and these studies offer the foundation for the synthesis of xylanbased materials. In recent years, xylan has been used for the preparation of food packaging materials, baking, papermaking, and food additives [21]. Oliveira has extracted xylan from corn cobs and found that xylan is a promising raw material for the pharmaceutical industry [25]. Because of the excellent performance, xylan-based materials are expected to be widely used in the medical field, especially for drug delivery and tissue engineering. A novel xylan/PVA hydrogel had been synthesized, and the study indicated that the gel was noncytotoxic and had a potential for biomedical application [26]. Recently, we prepared hemicellulose-based, 
$\mathrm{pH}$-sensitive, and biodegradable hydrogel for controlled drug delivery [22].

In this paper, the semi-interpenetrating network hydrogels based on xylan and poly(methacrylic acid) (xylan/ PMAA semi-IPN hydrogel) were synthesized and characterized. The chemical structure and surface morphology were analyzed using FT-IR and SEM, respectively. pH sensitivity, swelling reversibility, and swelling kinetics were also investigated. The exponential heuristic equation was used to analyze the swelling mechanism of the prepared hydrogels in different $\mathrm{pH}$ buffer solutions.

\section{Experimental}

2.1. Materials. The monomer methacrylic acid (MAA) was purchased from the Tianjin Kermel Chemical Reagent Company in China. The cross-linking agent N,N-methylenebisacrylamide (Bis), initiator ammonium persulfate (APS), and anhydrous sodium sulphite were purchased from the Tianjin Hongyan Chemical Reagent Factory in China. All of the reagents used were of analytical grade. Twice-distilled water was used for synthesis reactions and the preparation of buffer solutions.

2.2. Isolation of Xylan from Wheat Straw. Xylan was isolated from wheat straw by the following steps: wheat straw was first cut and dried in an oven at $60^{\circ} \mathrm{C}$ and then dewaxed using toluene and ethanol in a $2: 1$ volume ratio in a Soxhlet apparatus for $12 \mathrm{~h}$. After filtration, the residue was delignified using sodium chlorite in an acidic solution $(\mathrm{pH}$ 4.0) at $75^{\circ} \mathrm{C}$ for $2 \mathrm{~h}$, in order to obtain holocellulose. The holocellulose was treated using $10 \% \mathrm{KOH}$ at a 1:20 solid to liquid ratio at room temperature for $10 \mathrm{~h}$ and then filtered to separate the hemicellulose and cellulose. The filtrate was neutralized to $\mathrm{pH} 5.5$, concentrated under a reduced pressure, and then precipitated into 3 volumes of ethanol to obtain hemicellulosic powder.

To prepare pure xylan from wheat straw hemicellulose, the obtained hemicellulosic powders were treated using $0.05 \mathrm{~mol} / \mathrm{L} \mathrm{HCl}$ at a $1: 30$ solid to liquid ratio at $50^{\circ} \mathrm{C}$ for $2 \mathrm{~h}$. The obtained solution was neutralized to $\mathrm{pH} 5.5$ and concentrated under a reduced pressure and then precipitated in 3 volumes of ethanol. Pure xylan powders were finally obtained for further use. The prepared xylan contained 93.0\% xylose and 5.0\% arabinose (related to the total sugar content), which was determined using gas chromatography.

\subsection{Preparation of Xylan/PMAA Semi-IPN Hydrogels. Var-} ious amounts of xylan, MAA, and Bis were dissolved in distilled water, and the total concentration of both xylan and MAA was 20 wt.\%. Next, 1 wt.\% of APS and anhydrous sodium sulphite that related to the amount of methacrylic acid were added. After mixing, the mixture was sealed and placed in a water bath at $60^{\circ} \mathrm{C}$ for $2 \mathrm{~h}$ without stirring. After the gelation was achieved, the hydrogels were removed and cut into uniform sized pieces, and the cut samples were soaked in distilled water for $24 \mathrm{~h}$. During this period, it was necessary to change the water regularly for washing away the unreacted monomer and initiator. Finally, the hydrogels
TABLE 1: Feed compositions for the preparation of xylan/PMAA semi-IPN hydrogels.

\begin{tabular}{lcccc}
\hline \multirow{2}{*}{ Component } & \multicolumn{4}{c}{ Sample code } \\
& PMAA & Semi-IPN2 & Semi-IPN5 & Semi-IPN7 \\
\hline MAA (g) & 2.0 & 1.8 & 1.5 & 1.3 \\
Xylan (g) & 0 & 0.2 & 0.5 & 0.7 \\
Bis (g) & 0.040 & 0.036 & 0.030 & 0.024 \\
\hline
\end{tabular}

were vacuum-dried in an oven at $60^{\circ} \mathrm{C}$ for $24 \mathrm{~h}$. The feed composition for hydrogel preparation is presented in Table 1.

2.4. Characterizations. The dried hydrogel samples were analyzed using Fourier transform infrared spectroscopy within the frequency range of $4000-400 \mathrm{~cm}^{-1}$ after these samples were milled and mixed with $\mathrm{KBr}$ to press discs. The morphology of the hydrogels was observed using scanning electron microscope after swollen hydrogel samples were freeze-dried at $-50^{\circ} \mathrm{C}$ and coated with gold.

2.5. $\mathrm{pH}$ Sensitivity and Swelling Reversibility. $\mathrm{pH}$ sensitivity of the prepared hydrogels was determined gravimetrically by measuring the swelling extent of the hydrogels in buffer solutions of different $\mathrm{pH}(2.0-10.0)$ value at room temperature. The ionic strengths of buffer solutions were adjusted to $0.05 \mathrm{M}$ using $\mathrm{NaCl}$. To ensure complete swelling, samples were allowed to swell for $48 \mathrm{~h}$. The swollen hydrogels were weighed after the excess surface water was removed using a filter paper. The equilibrium swelling ratio was calculated using the following equation:

$$
S_{e}\left(\frac{g}{g}\right)=\frac{\left(W_{e}-W_{d}\right)}{W_{d}}
$$

where $W_{e}(g)$ is the weight of the swollen hydrogel at equilibrium state; $W_{d}(g)$ is the weight of the dry hydrogel.

The hydrogels swollen in buffer solution of $\mathrm{pH}=7.4$ were placed into the $\mathrm{pH} 2.0$ buffer solution. The hydrogels were removed and weighed after certain time intervals. The reswelling behaviors of the shrinking hydrogels were studied in $\mathrm{pH} 7.4$ buffer solution. At predetermined time interval, the hydrogels were weighed after blotting the excess water on the surface of the hydrogels. The swelling and deswelling tests were carried out repeatedly with a period of $48 \mathrm{~h}$. The swelling ratio at time $t$ was defined from the following equation:

$$
S_{t}\left(\frac{g}{g}\right)=\frac{\left(W_{t}-W_{d}\right)}{W_{d}}
$$

where $W_{t}$ is the weight of the hydrogel at swelling time $t$ and $W_{d}$ is defined as stated above.

2.6. Dynamic Swelling Kinetics. The known mass of hydrogel samples was placed in $100 \mathrm{~mL}$ buffer solutions at room temperature. After certain time interval, the hydrogels were removed and weighed after removing the excess water on the surface. The swelling ratio at time $t$ was calculated by (2). 


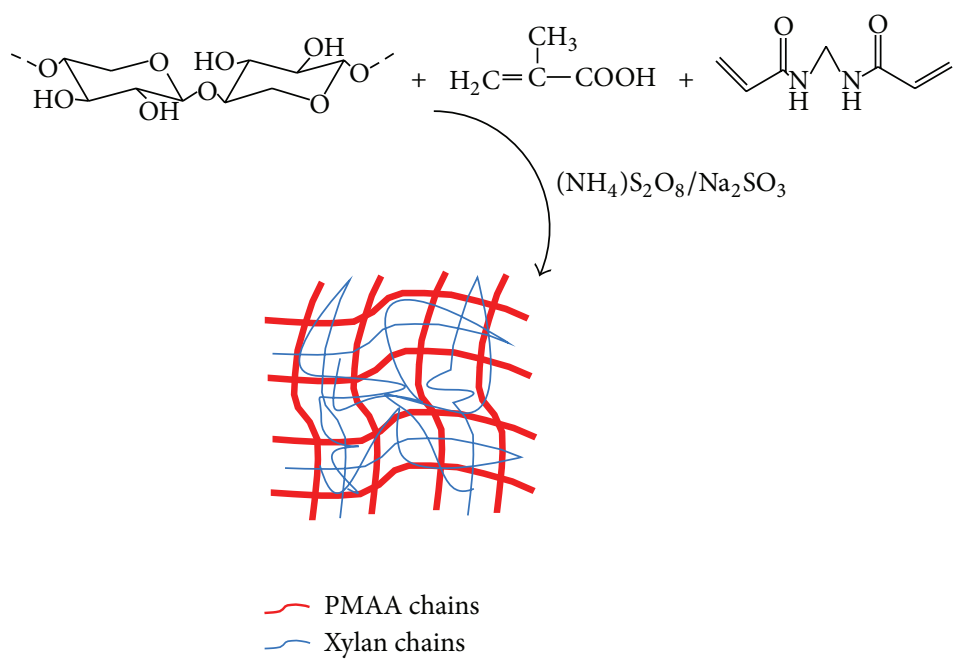

FIGURE 1: The proposed mechanism for the synthesis of xylan/PMAA semi-IPN hydrogel.

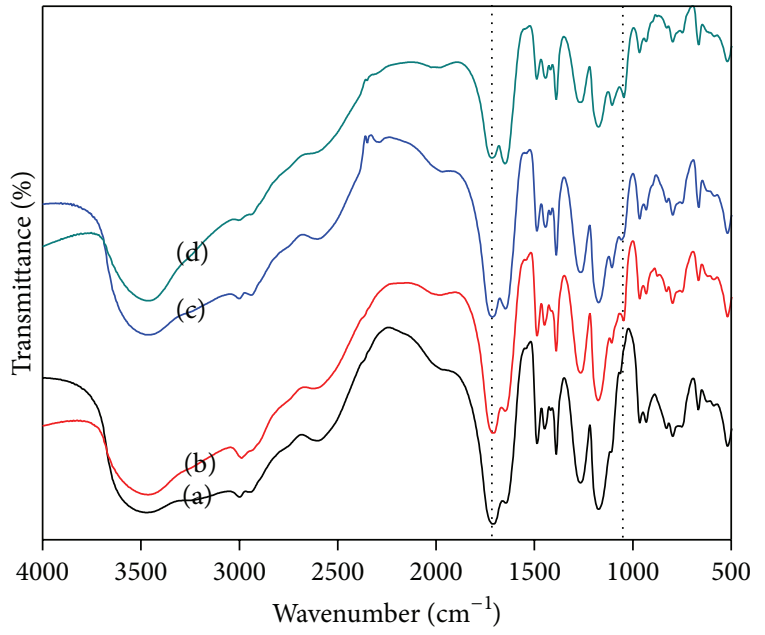

FIgURE 2: FT-IR spectra of PMAA (a), semi-IPN2 (b), semi-IPN5 (c), and semi-IPN7 (d).

\section{Results and Discussion}

3.1. Preparation of Xylan/PMAA Semi-IPN Hydrogels. The proposed mechanism for the synthesis of xylan/PMAA semiIPN hydrogels is shown in Figure 1. The redox initiator system, APS-anhydrous sodium sulphite, generated sulfate radical anion $\left(\mathrm{SO}_{4}{ }^{--}\right)$from APS, which initiated the polymerization of MAA, and poly(methacrylic acid) (PMAA) would be cross-linked by cross-linker Bis to form the threedimensional hydrogel. Xylan was incorporated into the threedimensional network of PMAA hydrogel mainly with hydrogel bonds formed through the hydroxyl groups and carbonyl groups on xylan and PMAA chains. Xylan/PMAA semi-IPN hydrogel was obtained as above.

3.2. FT-IR Analysis of Xylan/PMAA Semi-INP Hydrogels. FTIR spectra of semi-IPN2, semi-IPN5, semi-IPN7, and PMAA hydrogels are shown in Figure 2. The two absorption bands at
3430 and $2930 \mathrm{~cm}^{-1}$ were assigned to the stretching of $\mathrm{O}-\mathrm{H}$ and the stretching of $\mathrm{C}-\mathrm{H}$, respectively. The absorption bands at $1710 \mathrm{~cm}^{-1}, 1390 \mathrm{~cm}^{-1}$, and $965 \mathrm{~cm}^{-1}$ were the characteristic absorptions of $-\mathrm{COOH}$ groups, which are attributed to the $\mathrm{C}=\mathrm{O}$ stretching, the $\mathrm{C}-\mathrm{O}$ stretching vibration, and the $\mathrm{OH}$ in-plane vibration of carboxyl groups [27, 28]. The band at $1487 \mathrm{~cm}^{-1}$ was assigned to the bending vibration of $\mathrm{C}-\mathrm{H}$ of methyl groups [29]. The characteristic absorption peak for typical xylan was located at $1044 \mathrm{~cm}^{-1}$, which was attributed to the $\mathrm{C}-\mathrm{O}, \mathrm{C}-\mathrm{C}$ stretching, and the glycosidic linkage $v(\mathrm{C}-\mathrm{O}-\mathrm{C})$ contributions [30]. The sharp absorption peak at $894 \mathrm{~cm}^{-1}$ was assigned to $\mathrm{C} 1$ group frequency of vibration and frequency of vibration of the ring, which was the characteristic absorption of the glycosidic bond between sugar units [30]. More importantly, the intensity of the peak at $1044 \mathrm{~cm}^{-1}$ was enhanced and that of the peak at $1710 \mathrm{~cm}^{-1}$ declined with an increase of the content of xylan.

3.3. Morphology Analysis of Xylan/PMAA Semi-IPN Hydrogels. The morphology of the hydrogels prepared using different feed compositions is shown in Figure 3. The morphology of PMAA hydrogel presented a porous structure, which is typical morphology of PMAA-based hydrogel. The porosity of the xylan/PMAA semi-IPN hydrogels evidently increased and the pore wall became thin, and this resulted in the formation of the interconnected porous channel. The interconnected porous channels provide paths for the diffusion of water molecules, which is important to improve the sensitivity and swelling ratio of the hydrogel. The porous structure of the xylan/PMAA semi-IPN hydrogel would extend its application in some fields, such as wastewater treatment and the drug release control.

3.4. $p H$ Sensitivity of Xylan/PMAA Semi-IPN Hydrogels. The effect of $\mathrm{pH}$ on the equilibrium swelling ratio is presented in Figure 4. The equilibrium swelling ratio increased at first and then decreased when the $\mathrm{pH}$ value increased. This is primarily due to - $\mathrm{COOH}$ groups in hydrogel, and the dissociation of the 


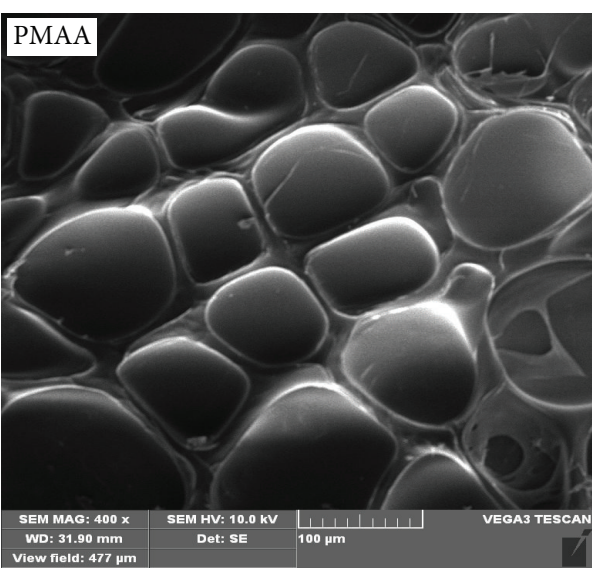

(a)

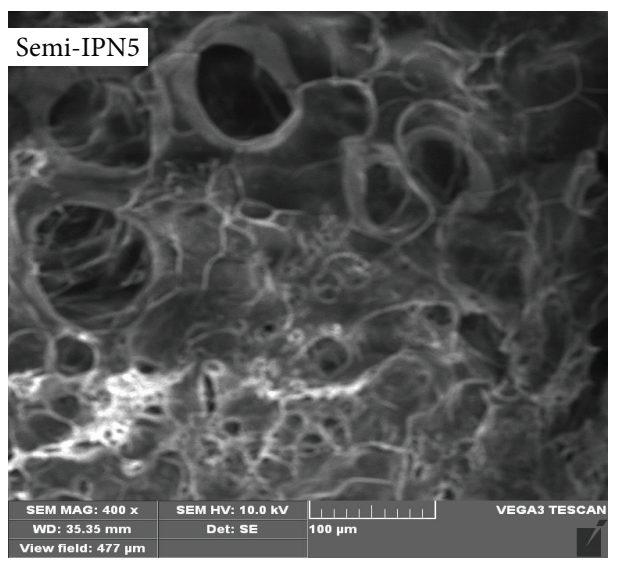

(c)

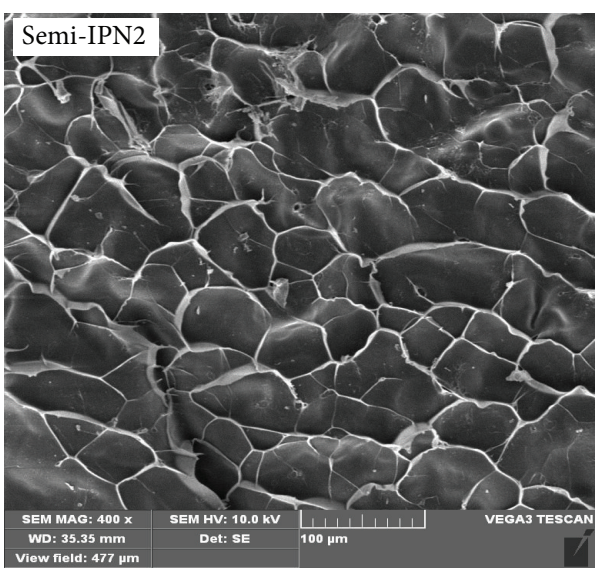

(b)

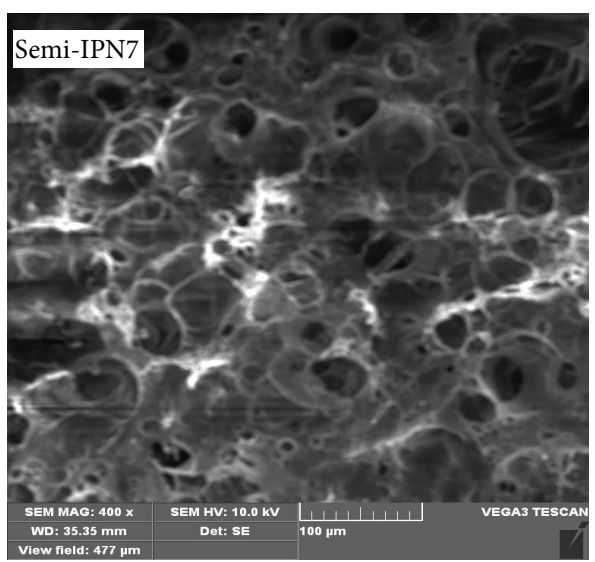

(d)

Figure 3: SEM images of PMAA (a), semi-IPN2 (b), semi-IPN5 (c), and semi-IPN7 (d).

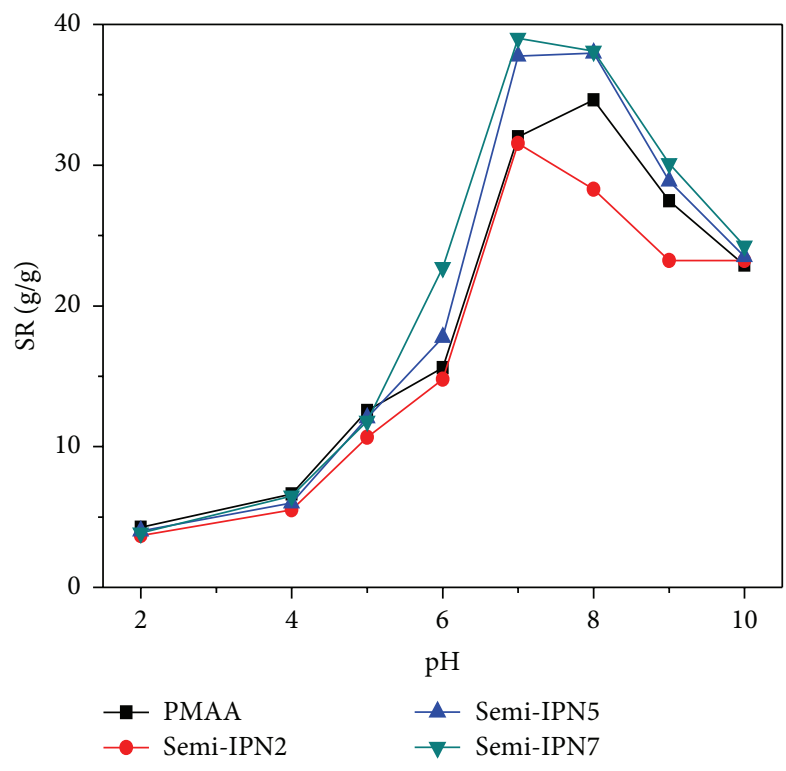

Figure 4: The swelling ratio of the hydrogels in different $\mathrm{pH}$ value buffer solutions.
- $\mathrm{COOH}$ group did not occur in a strongly acidic condition $\left(\mathrm{pH}<\mathrm{p} K_{\mathrm{a}}\right)$, and there were strong hydrogen bonds present within the hydrogels. When $\mathrm{pH}$ value is higher than $\mathrm{p} K_{\mathrm{a}}$ of the carboxyl group (4.28), the - $\mathrm{COOH}$ group changed into $-\mathrm{COO}^{-}$group which enhanced the electrostatic repulsion forces in the hydrogels network; therefore, the swelling ratio increased. But the interactions between the carboxylic acid anions and cations occurred with an increase of $\mathrm{pH}$ value, resulting in a slight decrease of the swelling ratio [16].

$\mathrm{pH}$ sensitivity is closely related to the morphology of the swollen hydrogels. To clearly analyze the sensitivity of the xylan/PMAA hydrogels, the surface morphology of the semi-IPN7 was observed, and the SEM micrographs are shown in Figure 5. As shown in Figure 5(a), the surface of the semi-IPN7 swollen in $\mathrm{pH} 2.0$ buffer solutions presented a little porous structure and the interconnected porous channels could not be observed, and this observation could be attributed to the hydrogen bond's effect in acidic solution. Meanwhile Figures 5(b) and 5(c) showed obviously the porous structure and the interconnected porous channels, which may be due to the higher swelling ratio in neutral and alkaline solutions. The porous size of the hydrogel sample 


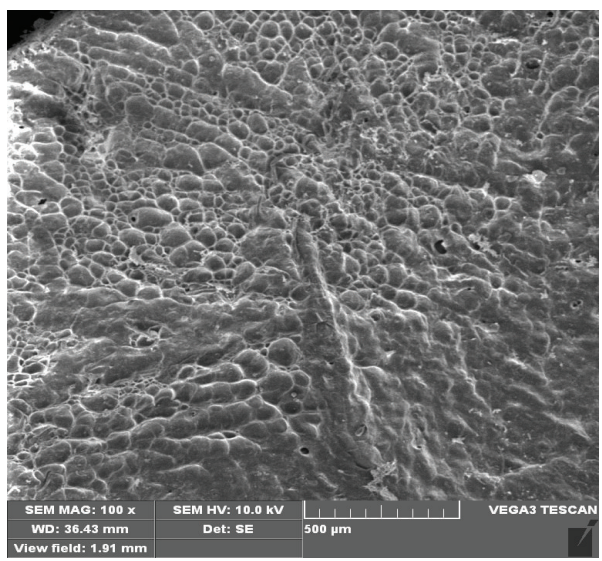

(a)

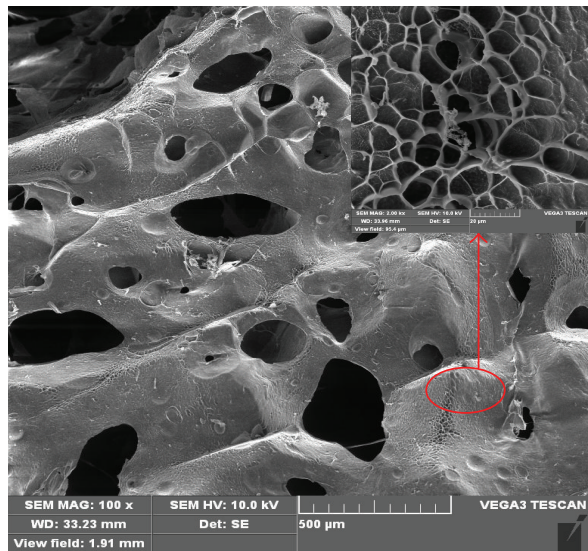

(b)

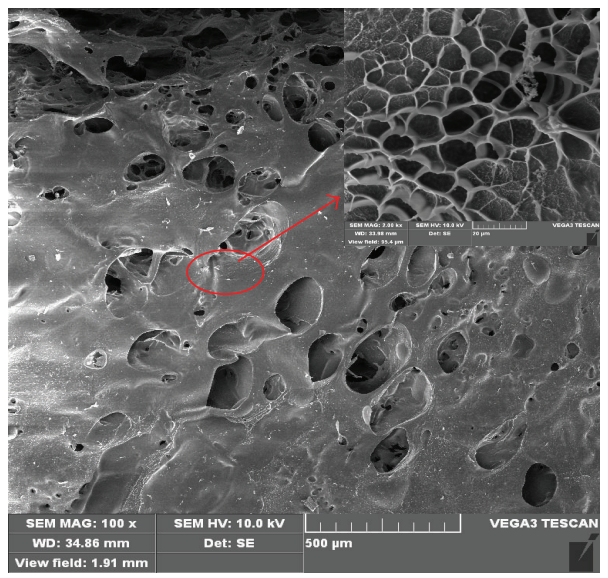

(c)

FIGURE 5: SEM images of the semi-IPN7 swollen in pH 2.0 (a), pH 7.4 (b), and pH 10.0 (c) buffer solutions.

swollen in $\mathrm{pH} 10.0$ solutions was smaller than that of the hydrogel sample swollen in $\mathrm{pH} 7.4$ solutions, and this could be attributed to the change of the swelling ratio when the $\mathrm{pH}$ value was increased, and the swelling behavior of the hydrogel in alkali solution was restrained because of the electrostatic screen effect. In addition, the pore density of the hydrogel sample swollen in $\mathrm{pH} 2.0$ and $\mathrm{pH} 7.4$ buffer solutions was less than that of the hydrogel sample swollen in $\mathrm{pH} 10.0$ buffer solution. To analyze this phenomenon, the nonporous region of the hydrogel sample in Figures 5(b) and 5(c) was magnified by 2000 times, and the wealth of porous structure was observed. Because - $\mathrm{COOH}$ groups would dissociate into $-\mathrm{COO}^{-}$in a higher $\mathrm{pH}\left(\mathrm{pH}>\mathrm{p} K_{\mathrm{a}}\right)$ solution, which could generate the electrostatic attraction forces with cations and reduce the repulsion force in hydrogel network, the porous size of the hydrogel sample decreased. Through the analysis of the morphology of the hydrogel, it is found that the trend of the pore size and the swelling degree change with $\mathrm{pH}$ change was the same.

3.5. Swelling Reversibility of Xylan/PMAA Semi-IPN Hydrogels. Figure 6 presents the swelling reversibility of the

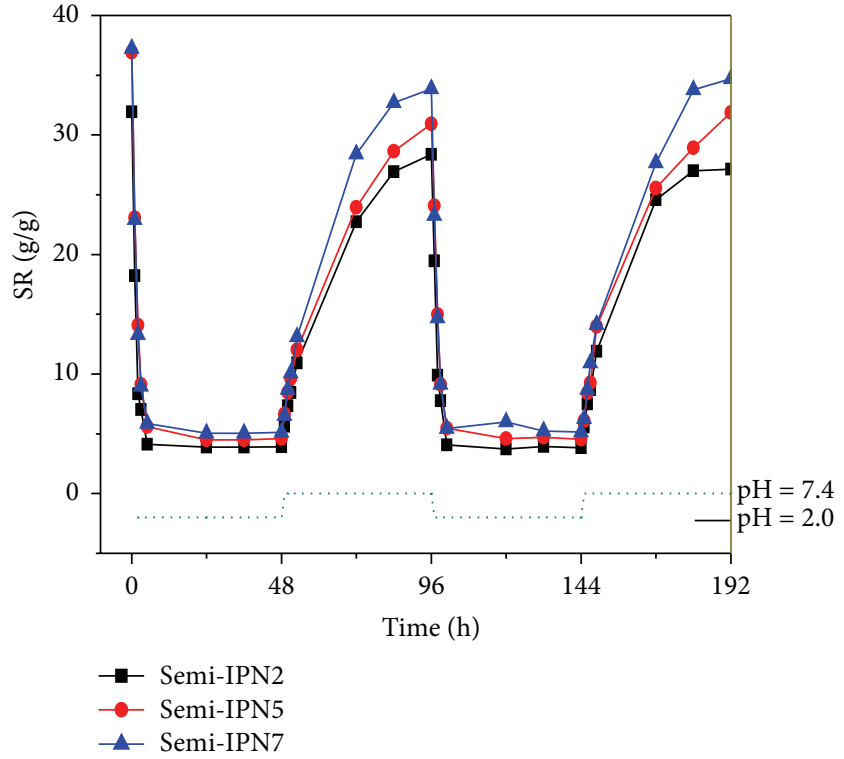

FIGURE 6: Swelling reversibility of the xylan/PMAA semi-IPN hydrogels in $\mathrm{pH} 2.0$ and $\mathrm{pH} 7.4$ buffer solutions. 

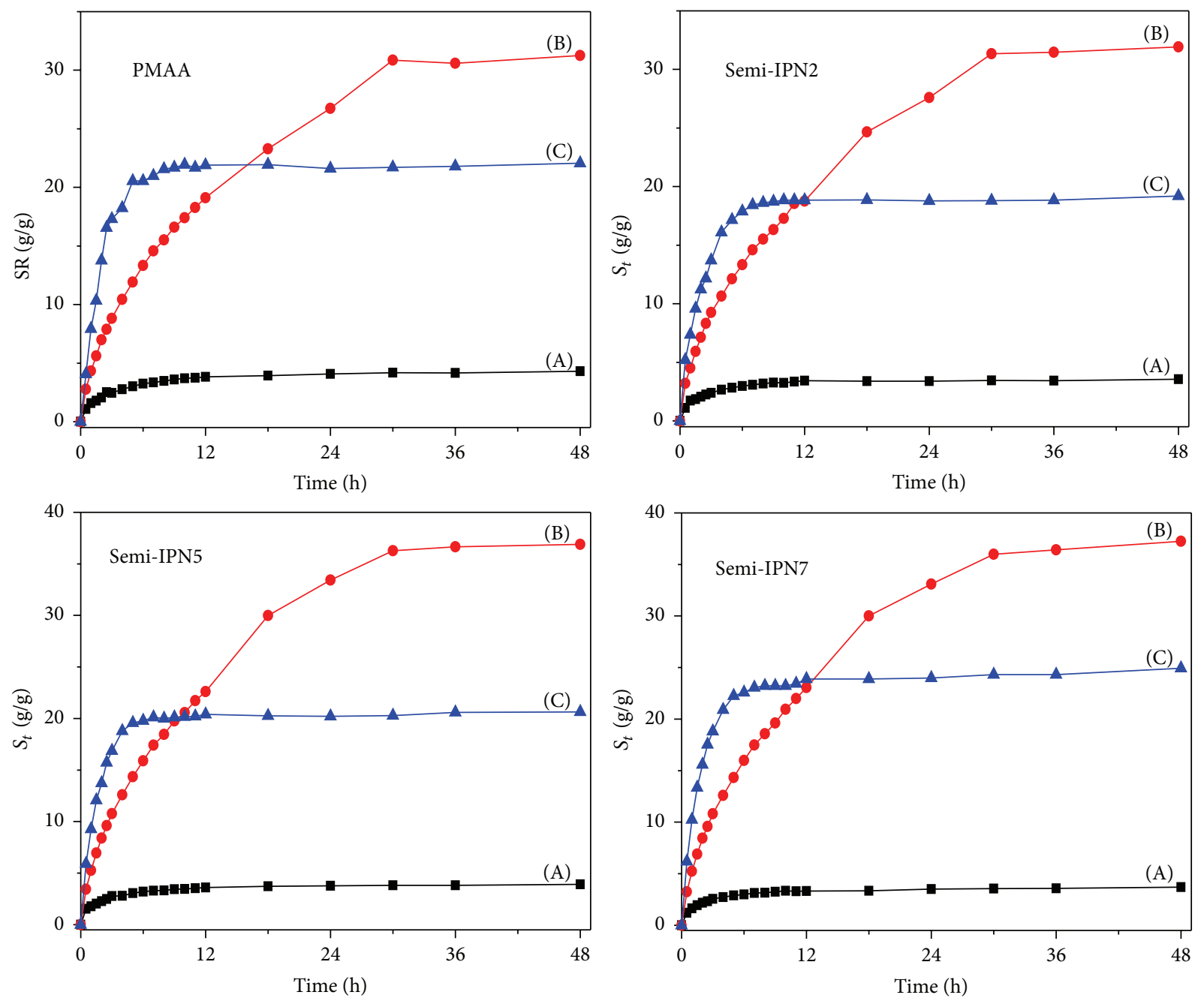

FIGURE 7: Time-dependent swelling ratio of the hydrogels in buffer solutions of pH 2.0 (A), 7.4 (B), and 10 (C).

xylan/PMAA semi-IPN hydrogels in buffer solutions of $\mathrm{pH}$ 2.0 and $\mathrm{pH}$ 7.4. The hydrogel samples swollen in $\mathrm{pH} 7.4$ buffer solution were placed into $\mathrm{pH} 2.0$ buffer solution and then transferred into the $\mathrm{pH} 7.4$ buffer solution. Samples rapidly shrank in $\mathrm{pH} 7.4$ buffer solution and reached the equilibrium. But the duration of the swelling was longer than the deswelling duration in $\mathrm{pH} 7.4$ buffer solution. The swelling-deswelling studies revealed that the swellingdeswelling behaviors of the xylan/PMAA semi-IPN hydrogels had excellent reversibility.

3.6. Dynamic Swelling Kinetics of Xylan/PMAA Hydrogels. The ionization degree of $-\mathrm{COOH}$ groups is different in various $\mathrm{pH}$ solutions, and this could result in the difference of hydrogen bond's effect in hydrogels, and hydrogels would have different swelling process in various buffer solutions. Figure 7 presents the time-dependent swelling ratio of the PMAA hydrogel and xylan/PMAA semi-IPN hydrogel in buffer solutions of pH 2.0, 7.4, and 10.0. As shown in Figure 7, the hydrogels prepared with different components exhibited similar dynamic swelling curves and swelling behavior on $\mathrm{pH}$ sensitivity. In the initial swelling process, the water adsorption rate of the hydrogels increased with a rise of $\mathrm{pH}$ value of buffer solutions, and this is related to the morphology of the hydrogels in buffer solution. The hydrogels swollen in $\mathrm{pH} 7.4$ buffer solution had the maximum swelling ratio and needed the longest time for achieving swelling equilibrium. The water adsorption rate of the hydrogels swollen in $\mathrm{pH} 10.0$ buffer solution was obviously higher than that of the hydrogels swollen in pH 2.0 and 7.4 solutions. According to the Donnan equilibrium theory, the swelling behavior of the hydrogel is due to the difference of the free-ion concentration osmotic pressure in inside and outside of the hydrogel. When the $\mathrm{pH}$ value of the buffer solution was high, the concentration of $\mathrm{COO}^{-}$groups in the hydrogels increased, and this resulted in a higher osmotic pressure and electrostatic repulsion forces in the hydrogel; therefore, it led to faster swelling rate of the hydrogels. However, the electrostatic repulsion forces decreased because of the swelling of the hydrogels, which is attributed to the charge screening effect by cations in buffer solution, and the hydrogels rapidly absorbed water in $\mathrm{pH} 10.0$ buffer media and achieved the swelling equilibrium. 
TABLE 2: Kinetic parameters of the PMAA hydrogel and xylan/PMAA semi-IPN hydrogels in buffer solutions of $\mathrm{pH}$ $2.0,7.4$, and 10 .

\begin{tabular}{lcccccc}
\hline \multirow{2}{*}{ Sample } & \multicolumn{2}{c}{$\mathrm{pH} 2.0$} & \multicolumn{2}{c}{$\mathrm{pH} 7.4$} & \multicolumn{2}{c}{$\mathrm{pH} \mathrm{10.0}$} \\
& $n$ & $R^{2}$ & $n$ & $R^{2}$ & $n$ & $R^{2}$ \\
\hline PMAA & 0.471 & 0.976 & 0.610 & 0.998 & 0.903 & 0.994 \\
Semi-IPN2 & 0.438 & 0.922 & 0.568 & 0.997 & 0.561 & 0.995 \\
Semi-IPN5 & 0.279 & 0.963 & 0.549 & 0.998 & 0.649 & 0.999 \\
Semi-IPN7 & 0.425 & 0.999 & 0.609 & 0.996 & 0.702 & 0.998 \\
\hline
\end{tabular}

Because of the excellent swelling property, the xylan/ PMAA semi-IPN hydrogels are expected to be a good carrier for colon-specific delivery. As the $\mathrm{pH}$ values in the human stomach and the colon are 2.0 and 7.0, respectively [31], the hydrogels, as drug carriers for colon-specific delivery, not only reduce the drug release in the gastric juice but also have high release efficiency and control release properties in the intestinal fluid.

The studies on the dynamic swelling kinetics of the hydrogels are important for their applications, especially for the drug release. To clearly understand the swelling mechanism of the hydrogels, the exponential heuristic equation was used to study the diffusion mechanism of water molecule in hydrogel network. It can be described as the following equation [32]:

$$
F=\frac{W}{W_{\infty}}=k t^{n} .
$$

Equation (3) can be deformed into the following equation:

$$
\ln \frac{W}{W_{\infty}}=\ln k+n \ln t
$$

where $F$ is the fractional uptake at time $t(\mathrm{~min}) ; k$ is constant incorporating characteristics of the polymer network and the solvent, whereas $n$ is the diffusion exponent which is indicative of the transport mechanism. The value of $n$ indicates the swelling mechanism of the hydrogels: the swelling process follows Fick's law when $n$ is less than 0.5 ; when $0.5<n<1$, it belongs to non-Fick's law, which is the result of the combined effects of solvent diffusion and relaxation of macromolecular chains; the swelling process is mainly decided by the relaxation of macromolecular chains when $n$ is greater than $1[3,4,32]$.

The diffusion exponent $n$ obtained from the profile of $\ln F-\ln t$ is listed in Table 2, where $n$ indicated the slope of curve. As can be seen from Table 2, the exponent $n$ trended to increase with a rise of $\mathrm{pH}$ value, and Fickian behavior was observed in $\mathrm{pH} 2.0$ buffer solution, and the swelling behavior in $\mathrm{pH} 7.4$ and 10.0 buffer solutions followed nonFickian behavior. The data of diffusion exponent $n$ of the xylan/PMAA semi-IPN hydrogels was lower than that of the PMAA hydrogels. As drug carriers for colon-specific delivery, the lower $n$ value is an advantage for the hydrogels, which not only reduces the drug release in the gastric juice but also could achieve the controlled release for drug in the intestinal fluid. These results indicated that the xylan/PMAA semi-IPN hydrogels can be considered a potential carrier for colonspecific and sustained drug release applications.

\section{Conclusions}

The xylan/PMAA semi-IPN hydrogels were fabricated successfully from xylan and methacrylic acid using N,Nmethylenebisacrylamide as cross-linker. The SEM images demonstrated the existence of a porous honeycomb-like structure in the hydrogels. The swelling kinetic experiments showed that Fickian behavior was observed in $\mathrm{pH} 2.0$ buffer solution. The swelling behavior of hydrogels in $\mathrm{pH} 7.4$ and 10.0 buffer solutions followed non-Fickian behavior which demonstrated that the water transport was controlled by hydrogel relaxation and water diffusion in $\mathrm{pH} 7.4$ and 10.0 buffer solutions.

Xylan plays a significant role in the morphology and swelling ratio of the prepared hydrogels, and the interconnected porous channels increased when the content of xylan was increased. The studies on $\mathrm{pH}$ sensitivity and dynamic swelling kinetics of hydrogels revealed that the semi-IPN xylan/PMAA hydrogels may serve as potential carriers for colon-specific and sustained drug release applications.

\section{Competing Interests}

The authors declare that they have no competing interests.

\section{Acknowledgments}

The authors appreciate the support by the National Natural Science Foundation of China (no. 20707016), the Fundamental Research Funds for the Central Universities (3102015BJ026), and the Seed Foundation of Innovation and Creation for Graduate Students in Northwestern Polytechnic University (Z2016169).

\section{References}

[1] Z. Peng and F. Chen, "Synthesis and properties of temperaturesensitive hydrogel based on hydroxyethyl cellulose," International Journal of Polymeric Materials and Polymeric Biomaterials, vol. 59, no. 6, pp. 450-461, 2010.

[2] S. Yang, S. Fu, Y. Zhou, C. Xie, and X. Li, "Preparation and release properties of a $\mathrm{pH}$-tunable carboxymethyl cellulose hydrogel/methylene blue host/guest model," International Journal of Polymeric Materials and Polymeric Biomaterials, vol. 60, no. 1, pp. 62-74, 2010.

[3] B. B. Mandal, S. Kapoor, and S. C. Kundu, "Silk fibroin/ polyacrylamide semi-interpenetrating network hydrogels for controlled drug release," Biomaterials, vol. 30 , no. 14, pp. $2826-$ 2836, 2009.

[4] Y. Huang, H. Yu, and C. Xiao, "pH-sensitive cationic guar gum/ poly (acrylic acid) polyelectrolyte hydrogels: swelling and in vitro drug release," Carbohydrate Polymers, vol. 69, no. 4, pp. 774-783, 2007.

[5] A. Vashist, Y. K. Gupta, and S. Ahmad, "Interpenetrating biopolymer network based hydrogels for an effective drug delivery system," Carbohydrate Polymers, vol. 87, no. 2, pp. 14331439, 2012. 
[6] B.-L. Guo and Q.-Y. Gao, "Preparation and properties of a $\mathrm{pH} /$ temperature-responsive carboxymethyl chitosan/poly $(\mathrm{N}$ isopropylacrylamide)semi-IPN hydrogel for oral delivery of drugs," Carbohydrate Research, vol. 342, no. 16, pp. 2416-2422, 2007.

[7] E. M. D’Urso and G. Fortier, “Albumin-poly(ethylene glycol) hydrogel as matrix for enzyme immobilization: biochemical characterization of crosslinked acid phosphatase," Enzyme and Microbial Technology, vol. 18, no. 7, pp. 482-488, 1996.

[8] J. P. Schillemans, W. E. Hennink, and C. F. van Nostrum, "The effect of network charge on the immobilization and release of proteins from chemically crosslinked dextran hydrogels," European Journal of Pharmaceutics and Biopharmaceutics, vol. 76, no. 3, pp. 329-335, 2010.

[9] L. Pescosolido, W. Schuurman, J. Malda et al., "Hyaluronic acid and dextran-based semi-IPN hydrogels as biomaterials for bioprinting," Biomacromolecules, vol. 12, no. 5, pp. 1831-1838, 2011.

[10] L. S. Moreira Teixeira, J. Feijen, C. A. van Blitterswijk, P. J. Dijkstra, and M. Karperien, "Enzyme-catalyzed crosslinkable hydrogels: emerging strategies for tissue engineering," Biomaterials, vol. 33, no. 5, pp. 1281-1290, 2012.

[11] W. Wang, H. Wang, C. Ren et al., "A saccharide-based supramolecular hydrogel for cell culture," Carbohydrate Research, vol. 346, no. 8, pp. 1013-1017, 2011.

[12] S. K. Seidlits, C. T. Drinnan, R. R. Petersen, J. B. Shear, L. J. Suggs, and C. E. Schmidt, "Fibronectin-hyaluronic acid composite hydrogels for three-dimensional endothelial cell culture," Acta Biomaterialia, vol. 7, no. 6, pp. 2401-2409, 2011.

[13] Y. S. Jeon, J. Lei, and J.-H. Kim, "Dye adsorption characteristics of alginate/polyaspartate hydrogels," Journal of Industrial and Engineering Chemistry, vol. 14, no. 6, pp. 726-731, 2008.

[14] S. Ekici, Y. Işıkver, and D. Saraydın, "Poly(acrylamide-sepiolite) composite hydrogels: preparation, swelling and dye adsorption properties," Polymer Bulletin, vol. 57, no. 2, pp. 231-241, 2006.

[15] M. Dalaran, S. Emik, G. Güçlü, T. B. Îyim, and S. Özgümüş, "Study on a novel polyampholyte nanocomposite superabsorbent hydrogels: synthesis, characterization and investigation of removal of indigo carmine from aqueous solution," Desalination, vol. 279, no. 1-3, pp. 170-182, 2011.

[16] C. Chang, B. Duan, J. Cai, and L. Zhang, "Superabsorbent hydrogels based on cellulose for smart swelling and controllable delivery," European Polymer Journal, vol. 46, no. 1, pp. 92-100, 2010.

[17] A. K. Bajpai and A. Giri, "Water sorption behaviour of highly swelling (carboxy methylcellulose-g-polyacrylamide) hydrogels and release of potassium nitrate as agrochemical," Carbohydrate Polymers, vol. 53, no. 3, pp. 271-279, 2003.

[18] Q. Xu, W. Huang, L. Jiang, Z. Lei, X. Li, and H. Deng, "KGM and PMAA based $\mathrm{pH}$-sensitive interpenetrating polymer network hydrogel for controlled drug release," Carbohydrate Polymers, vol. 97, no. 2, pp. 565-570, 2013.

[19] A. Ebringerova and T. Heinze, "Xylan and xylan derivativesbiopolymers with valuable properties, 1 . Naturally occurring xylans structures, isolation procedures and properties," Macromolecular Rapid Communications, vol. 21, no. 9, pp. 542-556, 2000.

[20] A. Ebringerová, Z. Hromádková, and T. Heinze, "Hemicellulose," in Polysaccharides I, vol. 186 of Advances in Polymer Science, pp. 1-67, Springer, Berlin, Germany, 2005.

[21] R. C. Sun, X. F. Sun, and J. Tomkinson, "Hemicelluloses and their derivatives," in Hemicelluloses: Science and Technology, P.
Gatenholm and M. Tenkanen, Eds., vol. 864 of ACS Symposium Series, pp. 2-22, American Chemical Society, Washington, DC, USA, 2004.

[22] X.-F. Sun, H.-H. Wang, Z.-X. Jing, and R. Mohanathas, "Hemicellulose-based $\mathrm{pH}$-sensitive and biodegradable hydrogel for controlled drug delivery," Carbohydrate Polymers, vol. 92, no. 2, pp. 1357-1366, 2013.

[23] X. F. Sun, R. C. Sun, P. Flower, and M. S. Baird, "Extraction and characterization of original lignin and hemicelluloses from wheat straw," Journal of Agricultural and Food Chemistry, vol. 53, no. 4, pp. 860-870, 2005.

[24] X.-F. Sun, Z. Jing, P. Fowler, Y. Wu, and M. Rajaratnam, "Structural characterization and isolation of lignin and hemicelluloses from barley straw," Industrial Crops and Products, vol. 33, no. 3, pp. 588-598, 2011.

[25] E. E. Oliveira, A. E. Silva, T. N. Júnior et al., "Xylan from corn cobs, a promising polymer for drug delivery: production and characterization," Bioresource Technology, vol. 101, no. 14, pp. 5402-5406, 2010.

[26] S. Tanodekaew, S. Channasanon, and P. Uppanan, "Xylan/ polyvinyl alcohol blend and its performance as hydrogel," Journal of Applied Polymer Science, vol. 100, no. 3, pp. 1914-1918, 2006.

[27] A. F. A. Chimphango, W. H. Van Zyl, and J. F. Görgens, "In situ enzymatic aided formation of xylan hydrogels and encapsulation of horse radish peroxidase for slow release," Carbohydrate Polymers, vol. 88, no. 3, pp. 1109-1117, 2012.

[28] W. Liu, X. Liu, S. Zhu, X. Liu, and G. Han, "Ultrasonic synthesis of pH-sensitive PMAA hydrogel," Acta Chimica Sinica, vol. 70, no. 3, pp. 272-276, 2012.

[29] Y.-J. Li, X.-F. Sun, Q. Ye, B.-C. Liu, and Y.-G. Wu, "Preparation and properties of a novel hemicellulose-based magnetic hydrogel," Acta Physico-Chimica Sinica, vol. 30, no. 1, pp. 111-120, 2014.

[30] X. F. Sun, F. Xu, R. C. Sun, Z. C. Geng, P. Fowler, and M. S. Baird, "Characteristics of degraded hemicellulosic polymers obtained from steam exploded wheat straw," Carbohydrate Polymers, vol. 60, no. 1, pp. 15-26, 2005.

[31] M. A. Kabel, F. Carvalheiro, G. Garrote et al., "Hydrothermally treated xylan rich by-products yield different classes of xylooligosaccharides," Carbohydrate Polymers, vol. 50, no. 1, pp. 4756, 2002.

[32] C. Liu, Y. Chen, and J. Chen, "Synthesis and characteristics of $\mathrm{pH}$-sensitive semi-interpenetrating polymer network hydrogels based on konjac glucomannan and poly(aspartic acid) for in vitro drug delivery," Carbohydrate Polymers, vol. 79, no. 3, pp. 500-506, 2010. 

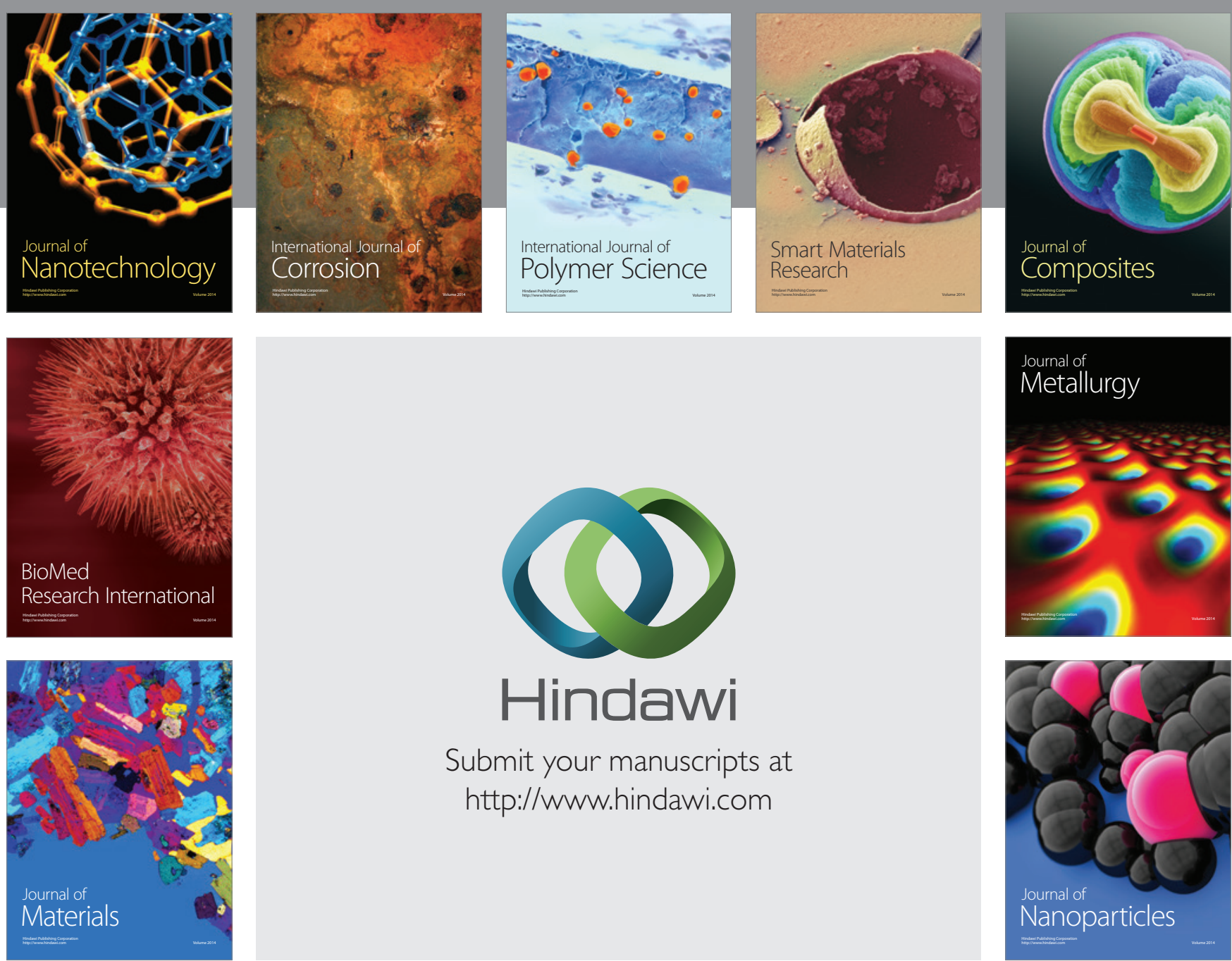

\section{Hindawi}

Submit your manuscripts at

http://www.hindawi.com

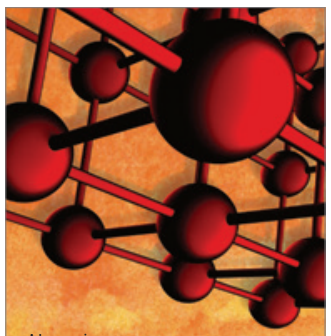

Materials Science and Engineering
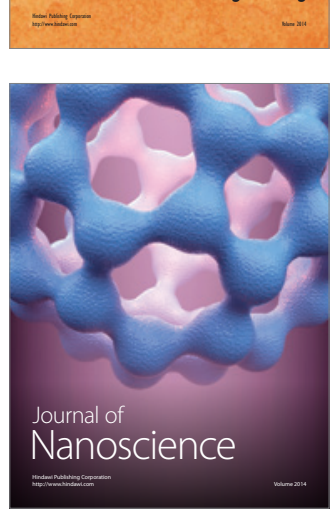
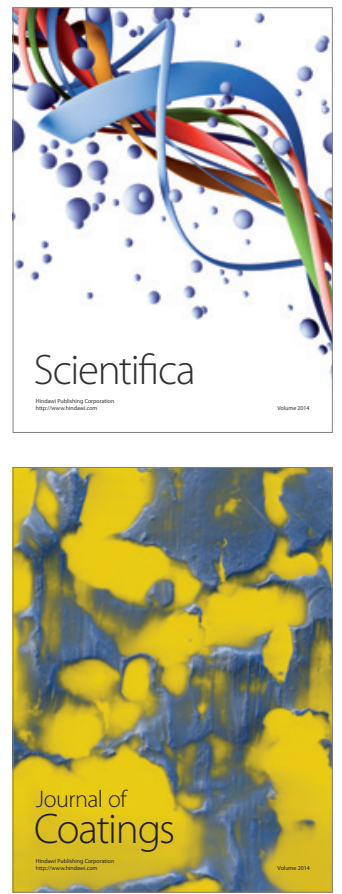
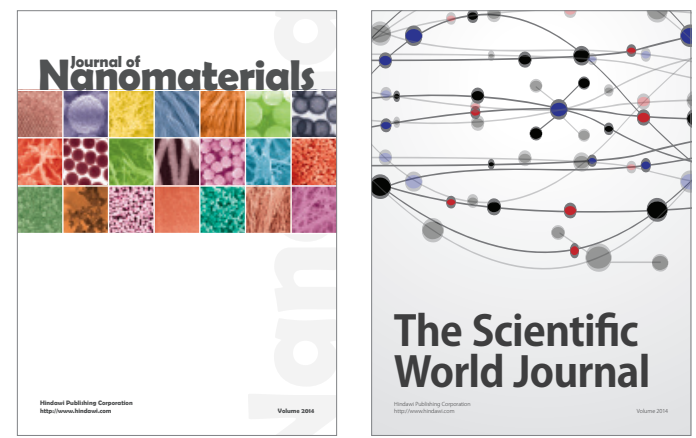

The Scientific World Journal
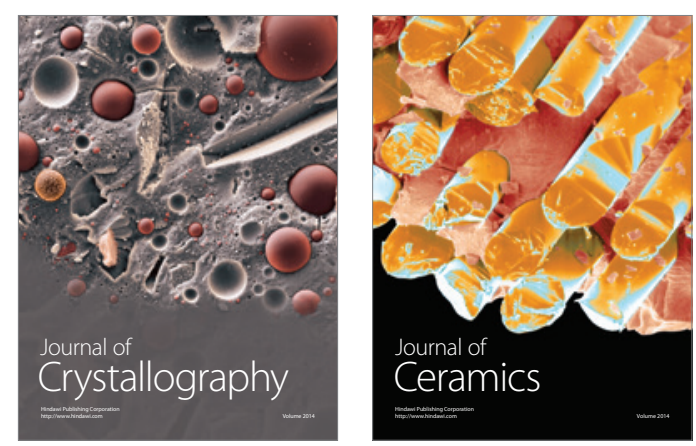
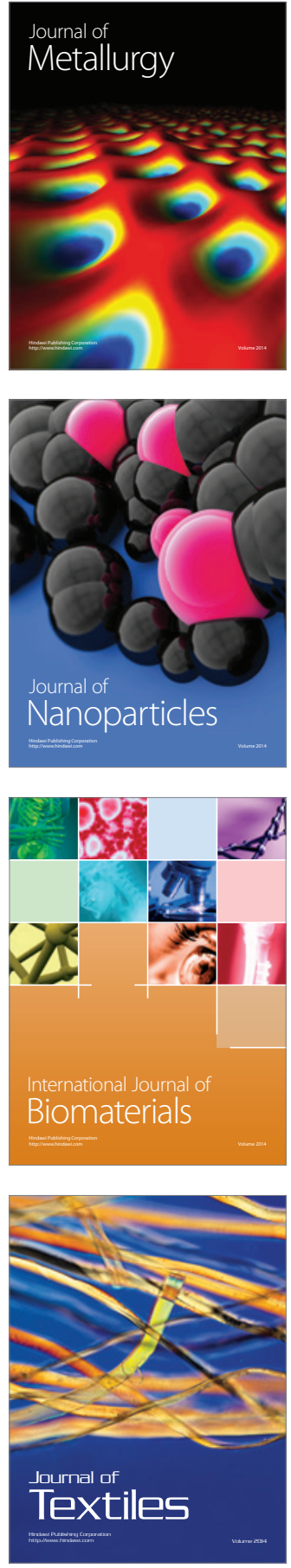